\title{
Construction of an Optimal Rest-to-Rest Control of a Rotating Beam
}

\author{
G. M. Sklyar • J. Woźniak
}

Received: 15 October 2012 / Accepted: 26 August 2014 / Published online: 5 September 2014 (C) The Author(s) 2014. This article is published with open access at Springerlink.com

\begin{abstract}
The paper is a continuation of our previous work on the controllability problems of a slowly rotating Timoshenko beam. The optimal control problem for this system is stated and the numerical method of obtaining its solution is given. Our approach is based upon the use of a special character of functions generating a corresponding moment problem.
\end{abstract}

Keywords Timoshenko beam $\cdot$ Moment problem $\cdot$ Rest-to-rest control $\cdot$ Optimal control

Mathematics Subject Classification $\quad 49 \mathrm{~J} 20 \cdot 30 \mathrm{E} 05 \cdot 35 \mathrm{~A} 35$

\section{Introduction}

We consider the model of a Timoshenko beam slowly rotating in a horizontal plane, where the left end of the beam is clamped to the disk of a driving motor [1]. A number of works concerning various controllability problems for the model were published. In particular, Krabs and Sklyar [2] analyzed the appropriate non-Fourier trigonometric moment problem in 1999, inspired by Russel (e.g., [3]). They showed that the system is rest-to-rest controllable, under some conditions on the physical properties of the materials of the beam, if the time of steering is strictly greater than the certain critical

Communicated by Günther Leugering.

G. M. Sklyar $(\bowtie) \cdot J$. Woźniak

Institute of Mathematics, University of Szczecin, ul. Wielkopolska 15, 70-451 Szczecin, Poland

e-mail: sklar@univ.szczecin.pl

J. Woźniak

e-mail:wozniak@univ.szczecin.pl 
value (minimal controllability time). The more general problem of description of all reachable states of the system of the beam and the disk was solved in $[4,5]$. Those results were used to obtain a new controllability condition of the beam in the form of smoothness of end states [6]. A similar model of a non-homogeneous beam was considered in [7] where the minimal time for a rest-to-rest controllability was found.

It is worth to mention that there exists only at most one control function steering from the preassigned position of rest to another, if the time of steering equals exactly the minimal value. If we allow the time to be greater than the minimal controllability one, then there exists an infinite number of such controls. Therefore, it is natural to consider only the intervals larger than the minimal time and searching for controls satisfying additional special requirements. The numerical construction of a control function steering the beam from one position of rest to another was given in 2000 for a time of movement large enough, in the form of a piecewise constant function [8]. In the work, we consider another problem. Namely, we want to construct a control that is optimal in the sense of minimal energy (i.e., fuel) consumption during the movement, steering the beam from one assigned position of rest to another, given the time is greater than the minimal controllability time. The idea of the framework is to observe that the optimal function lies in a closure of a specific linear span, which allows to rewrite the control as a solution of an appropriate moment problem. The moment problem obtained in this way is non-Fourier-the eigenvalues of a related operator are simple, however they come in pairs asymptotically close to each other. It still appears to be possible to approximate such pairs of related moment generating functions corresponding to close eigenvalues by some divided differences. Besides, those functions are close to periodic ones, and this fact allows us to approximate the moment problem by another infinite moment problem of a special type. Such an idea was used for solving a certain problem of optimal control of a string in [9]. In the end we obtain the finite set of linear equations. The approach of replacing an infinite set of equations of some non-Fourier moment problem by a finite set is used, for example, in $[10,11]$. A similar idea for a non-homogeneous string was presented lately in [12]. We also show that the speed of convergence of the presented method is essentially better than the one obtained in the classical truncation method [13].

\section{Optimal Control Problem as a Moment Problem}

We consider the linearized model from [1] of a Timoshenko beam slowly rotating in a horizontal plane. The left end of the beam is clamped to the disk of a driving motor. We denote by $r>0$ the radius of that disk and let $\theta=\theta(t)$ be the rotation angle considered as a function of time $(t \geq 0)$. Further on, we denote some of the physical properties of the beam: $E$ - the flexural rigidity, $K$-shear stiffness and $A$-cross section area; the length of the beam is assumed to be 1 . We denote by $w(x, t)$ the deflection of the center line of the beam and by $\xi(x, t)$ the rotation angle ${ }^{1}$ of the cross section area at the location $x$ and at the time $t$. Then the linearized model of behavior of $w$ and $\xi$ is given (see [14] and the references therein) in the form of the following

\footnotetext{
1 Note that some authors use another notation for the angle variable, namely $\psi=-\xi$.
} 
system of two partial differential equations:

$$
\begin{aligned}
& \ddot{w}(x, t)-w^{\prime \prime}(x, t)-\xi^{\prime}(x, t)=-\ddot{\theta}(t)(r+x), \\
& \ddot{\xi}(x, t)-\gamma^{2} \xi^{\prime \prime}(x, t)+w^{\prime}(x, t)+\xi(x, t)=\ddot{\theta}(t)
\end{aligned}
$$

with $x \in] 0,1\left[\right.$ and $t>0$, where $\gamma^{2}=\frac{E A}{K}, \dot{y}(x, t)=\frac{\partial}{\partial t} y(x, t), y^{\prime}(x, t)=\frac{\partial}{\partial x} y(x, t)$. In addition to (1), we impose boundary conditions given by

$$
w(0, t)=\xi(0, t)=w^{\prime}(1, t)+\xi(1, t)=\xi^{\prime}(1, t)=0
$$

for $t \geq 0$, which mean that there is no deformation at the clamped end and the energy balance law holds on the other end. We also assume that the radius $r$ of the disk is nonsingular [14]. The beam will be controlled by the angular acceleration of the motor disk, that is by $\ddot{\theta}$.

From now on we assume that $\gamma=1$. Let time $T>0$ (large enough) and position $\theta_{T} \in \mathbb{R}$ be given. We steer the beam by angular acceleration of the disk, that is by $\ddot{\theta}$. For our convenience let us denote $u(t)=\ddot{\theta}(T-t)$ and assume that $T=2 M, M \in \mathbb{N}$, $M>2$. We want to find a control $u \in L^{2}[0, T]$ such that it moves the beam from the position of rest at time $t=0$ and angle $\theta=0$, i.e.,

$$
\begin{aligned}
w(x, 0) & =\dot{w}(x, 0)=0, \\
\xi(x, 0) & =\dot{\xi}(x, 0)=0, \\
\theta(0) & =\dot{\theta}(0)=0,
\end{aligned}
$$

$x \in[0,1]$, to the position of rest at time $t=T$ and angle $\theta_{T}$, i.e.,

$$
\begin{aligned}
w(x, T) & =\dot{w}(x, T)=0, \\
\xi(x, T) & =\dot{\xi}(x, T)=0, \\
\dot{\theta}(T) & =0, \quad \theta(T)=\theta_{T},
\end{aligned}
$$

$x \in[0,1]$. Moreover, we want $u$ to be optimal in the following sense:

$$
\min _{u} \int_{0}^{T} u^{2}(t) \mathrm{d} t .
$$

It is shown [1] that if $T \geq 4$ the problem of finding a rest to rest control (1)-(4) is solvable, and the solution is a real function. It is proven that it is equivalent to solving the following moment problem

$$
\begin{aligned}
& \int_{0}^{T} \mathrm{e}^{i \sqrt{\lambda_{n}} t} u(t) \mathrm{d} t=0, \quad n \in \mathbb{Z}, \\
& \int_{0}^{T} u(t) \mathrm{d} t=0, \\
& \int_{0}^{T} t u(t) \mathrm{d} t=\theta_{T},
\end{aligned}
$$


where for $n>0, \lambda_{n}$ is an eigenvalue of the operator connected with the beam equation (1), and for $n \leq 0$ we put $\lambda_{-n}=-\lambda_{n+1}$. In [2] an analysis of values $\lambda_{n}$ is given, in particular it is shown that for $n>0$ we have

$$
\sqrt{\lambda_{n}}= \begin{cases}\frac{2 k-1}{2} \pi-\varepsilon_{n} & \text { for } n=2 k-1, \\ \frac{2 k-1}{2} \pi+\varepsilon_{n} & \text { for } n=2 k, \quad \varepsilon_{n}=\mathcal{O}\left(\frac{1}{n}\right) .\end{cases}
$$

The moment problem (6) has a major disadvantage - its generating family contains two families of exponential functions with exponents that approach one another, therefore it does not constitute a Riesz basis. We will work this out by considering an equivalent moment problem. It is easy to see that each pair of moment equations from (6), corresponding to the eigenvalues close to each other, that is

$$
\int_{0}^{T} \mathrm{e}^{i \sqrt{\lambda_{2 k-1}} t} u(t) \mathrm{d} t=0, \quad \int_{0}^{T} \mathrm{e}^{i \sqrt{\lambda_{2 k}} t} u(t) \mathrm{d} t=0,
$$

is equivalent to a pair of equations

$$
\int_{0}^{T} \mathrm{e}^{i \sqrt{\lambda_{2 k-1}} t} u(t) \mathrm{d} t=0, \quad \int_{0}^{T} \frac{\mathrm{e}^{i \sqrt{\lambda_{2 k}} t}-\mathrm{e}^{i \sqrt{\lambda_{2 k-1}} t}}{\sqrt{\lambda_{2 k}} t-\sqrt{\lambda_{2 k-1}} t} u(t) \mathrm{d} t=0,
$$

which allows us to rewrite the original moment problem (6) as

$$
\begin{aligned}
& \int_{0}^{T} \mathrm{e}^{i \sqrt{\lambda_{k}} t} u(t) \mathrm{d} t=0, \quad-N+1 \leq 2 k-1<N, \\
& \int_{0}^{T} \mathrm{e}^{i \sqrt{\lambda_{2 k-1}} t} u(t) \mathrm{d} t=0, \quad-N+1>2 k-1 \text { or } 2 k-1>N, \\
& \int_{0}^{T} \frac{\mathrm{e}^{i \sqrt{\lambda_{2 k}} t}-\mathrm{e}^{i \sqrt{\lambda_{2 k-1}}}}{\sqrt{\lambda_{2 k}} t-\sqrt{\lambda_{2 k-1}} t} u(t) \mathrm{d} t=0, \quad-N+1>2 k-1 \text { or } 2 k-1>N, \\
& \int_{0}^{T} u(t) \mathrm{d} t=0, \\
& \int_{0}^{T} t u(t) \mathrm{d} t=\theta_{T},
\end{aligned}
$$

for a fixed even integer $N$, with generating functions constituting a Riesz basis (see [5]).

\section{Classical Truncation Method}

In a general Hilbert (separable) space setting, solving an infinite moment problem of the form

$$
\left\langle u, z_{j}\right\rangle=c_{j}, \quad j \in \mathbb{N}
$$

(of which (8) is a special case), is not an easy task. One of the most common approaches consists in truncating the system into a finite number of equations (see, for example, [13] or the monograph [15]). Let us discuss it in details. 
Following the notation from [15], consider the truncated moment problem

$$
\left\langle u, z_{j}\right\rangle=c_{j}, \quad j \in\{1, \ldots, N\}
$$

for an arbitrary $N \in \mathbb{N}$. Providing the sequence $\left(z_{j}\right)$ is linearly independent, there exists exactly one solution $u^{N}$ of (10) of the form

$$
u^{N}:=\sum_{j=1}^{N} \xi_{j}^{N} z_{j}
$$

and $u^{N}$ has the smallest possible norm $\left\|u^{N}\right\|$ among all solutions of (10). Moreover, if we assume that

$$
\lim _{N \rightarrow \infty} \inf \{\|u\| \mid u \text { solves (10) }\}<\infty,
$$

then there is exactly one solution $u=u^{\infty}$ of (9) with the least norm, given by

$$
u^{\infty}:=\lim _{N \rightarrow \infty} u^{N}
$$

The truncating method has no bound though for the convergence speed of the solutions $u^{N}$ of (10) to the solution $u^{\infty}$ of (9), and this speed can be made arbitrarily slow. Let us consider a following example. Let $e_{0}, e_{1}, \ldots$ be an orthonormal basis, choose $z_{0} \neq e_{0}$ such that $\left\langle e_{0}, z_{0}\right\rangle=1$. Now consider a following moment problem:

$$
\left\langle u, e_{0}\right\rangle=1, \quad\left\langle u, \tilde{e}_{k}\right\rangle=0, \quad k \in \mathbb{N},
$$

where $\tilde{e}_{k}=e_{k}-\left\langle z_{0}, e_{k}\right\rangle e_{0}$. The generating family of (11), that is $e_{0}, \tilde{e}_{1}, \tilde{e}_{2}, \ldots$, is in fact a biorthogonal set to $z_{0}, e_{1}, e_{2}, \ldots$, therefore it constitutes a Riesz basis. One can easily observe that this system has a unique solution, namely $u^{\infty}=z_{0}$. Now consider a truncation of (11), that is

$$
\left\langle u, e_{0}\right\rangle=1, \quad\left\langle u, \tilde{e}_{k}\right\rangle=0, \quad k \in\{1, \ldots, N\} .
$$

Observe that for the solution $u^{N}$ of (12) holds the following:

$$
u^{N}=z_{0}+\sum_{k=N+1}^{\infty}\left\langle u^{N}, \tilde{e}_{k}\right\rangle e_{k}=z_{0}-\sum_{k=N+1}^{\infty}\left\langle z_{0}, e_{k}\right\rangle e_{k},
$$

so $u^{N}$ converges to $z_{0}$, but the speed of convergence is arbitrarily slow, depending on $z_{0}$.

In our case, a moment problem (8) is very similar to (11), we also need to find a vector $z_{0}$ biorthogonal to $e_{0}(t)=t$. We deal with a much more complicated set of generating vectors though, they are not biorthogonal to each other. The classical general methods, like truncation or Galerkin approximation, do not use any properties of 
a moment problem generating vectors. In case of not biorthogonal generating vectors, the resulting speed of convergence may be even less predictable. Here we propose another approach. The moment problem in question, (8), is generated by some special functions. We exploit the fact that the exponents $\sqrt{\lambda_{n}}$ have a special asymptotic behavior, given by (7). This means that those exponential functions are in fact close to trigonometric functions - at least for large indices. Knowing this we try not to truncate the problem, but to find the orthogonal complement subspace, and to seek for the approximation of the optimal solution in this subspace. The speed of convergence of the obtained approximations is significantly better than of the one obtained by general methods.

\section{Approximation of a Moment Problem}

In this section we show that the optimal solution of moment problem (8) can be approximated by a solution that is optimal in the sense of (5) of

$$
\begin{aligned}
& \int_{0}^{T} \mathrm{e}^{i \sqrt{\lambda_{n}} t} u(t) \mathrm{d} t=0, \quad\left\lfloor\frac{-N-2}{2}\right\rfloor \leq n \leq\left\lfloor\frac{N+1}{2}\right\rfloor, \\
& \int_{0}^{T} \mathrm{e}^{i \frac{2 n+1}{2} \pi t} u(t) \mathrm{d} t=0, \quad\left\lfloor\frac{-N-2}{2}\right\rfloor \geq n \text { or } n \geq\left\lfloor\frac{N+1}{2}\right\rfloor, \\
& \int_{0}^{T} t \mathrm{e}^{i \frac{2 n+1}{2} \pi t} u(t) \mathrm{d} t=0, \quad\left\lfloor\frac{-N-2}{2}\right\rfloor \geq n \text { or } n \geq\left\lfloor\frac{N+1}{2}\right\rfloor, \\
& \int_{0}^{T} u(t) \mathrm{d} t=0, \\
& \int_{0}^{T} t u(t) \mathrm{d} t=\theta_{T},
\end{aligned}
$$

where $N \in 2 \mathbb{N}$ is large enough ${ }^{2}$.

To do this we will use the notion of quadratically close Riesz bases and some results from [12], which we recollect here briefly.

We say that two families $\left(\varphi_{k}\right)$ and $\left(\varphi_{k}^{\prime}\right)$ of a separable Hilbert space are quadratically $\varepsilon$-close if

$$
\sum_{k}\left\|\varphi_{k}-\varphi_{k}^{\prime}\right\|^{2}<\varepsilon
$$

We will use the following two lemmas later, the first one is an essential theorem from [16].

Lemma 4.1 (cf. [16]) Assume $\left(\psi_{k}\right)$ and $\left(\psi_{k}^{\prime}\right)$ are Riesz bases biorthogonal to $\left(\varphi_{k}\right)$ and $\left(\varphi_{k}^{\prime}\right)$ respectively. If $\left(\varphi_{k}\right)$ and $\left(\varphi_{k}^{\prime}\right)$ are quadratically $\varepsilon$-close then $\left(\psi_{k}\right)$ and $\left(\psi_{k}^{\prime}\right)$ are quadratically $\varepsilon$-close.

\footnotetext{
$\overline{2 \text { From now on we will write " }|n|} \leq N$ " instead of " $\left\lfloor\frac{-N-2}{2}\right\rfloor \leq n \leq\left\lfloor\frac{N+1}{2}\right\rfloor$ " and " $|n|>N$ " instead of “ $\left\lfloor\frac{-N-2}{2}\right\rfloor \geq n$ or $n \geq\left\lfloor\frac{N+1}{2}\right\rfloor$ ” for simplicity.
} 
Next lemma was stated in [12].

Lemma 4.2 Denote by $\mathcal{L}$ and $\mathcal{L}_{N}$ the closures of linear spans of $\left(\varphi_{k}\right)$ and $\left(\varphi_{k}^{N}\right)$ respectively, and by $\mathcal{L}^{\perp}$ and $\mathcal{L}_{N}^{\perp}$ the orthonormal complements of $\mathcal{L}$ and $\mathcal{L}_{N}$. Let $\left(x_{k}\right)$ be the orthonormal basis of $\mathcal{L}^{\perp}$ and let $P: \mathcal{L}^{\perp} \rightarrow \mathcal{L}_{N}^{\perp}$ be a projection. There exist a positive constant $C$ such that for any sufficiently small $\varepsilon>0$ the systems $\left(x_{k}\right)$ and $\left(P x_{k}\right)$ are quadratically $C \varepsilon$-close, provided $\left(\varphi_{k}\right)$ and $\left(\varphi_{k}^{N}\right)$ are quadratically $\varepsilon$-close.

Proof Given $x \in \mathcal{L}^{\perp}$ we write $x=P x+Q x$, where $Q: \mathcal{L}^{\perp} \rightarrow \mathcal{L}_{N}$ is the projection. Then, because $\left(\varphi_{n}^{N}\right)$ is a Riesz basis, there exists a constant $C$ such that for any $y \in \mathcal{L}_{N}$ the inequality

$$
\|y\|^{2} \leq C \sum_{n}\left|\left\langle y, \varphi_{n}^{N}\right\rangle\right|^{2}
$$

holds [17] and because $\left(\varphi_{n}^{N}\right)$ is quadratically $\varepsilon$-close to $\left(\varphi_{n}\right)$, for sufficiently small $\varepsilon, C$ can be chosen universally for all $N \geq N_{0}$. Then we have

$$
\begin{aligned}
\sum_{k}\left\|x_{k}-P x_{k}\right\|^{2} & =\sum_{k}\left\|Q x_{k}\right\|^{2} \leq C \sum_{k, n}\left|\left\langle Q x_{k}, \varphi_{n}^{N}\right\rangle\right|^{2} \\
& =C \sum_{k, n}\left|\left\langle x_{k}, \varphi_{n}^{N}\right\rangle\right|^{2}=C \sum_{k, n}\left|\left\langle x_{k}, \varphi_{n}^{N}-\varphi_{n}\right\rangle\right|^{2} .
\end{aligned}
$$

Because $\left(x_{k}\right)$ constitutes an orthonormal set, the last expression is less or equal to

$$
C \sum_{n}\left\|\varphi_{n}^{N}-\varphi_{n}\right\|^{2}
$$

It follows that

$$
\sum_{k}\left\|x_{k}-P x_{k}\right\|^{2} \leq C \sum_{n}\left\|\varphi_{n}^{N}-\varphi_{n}\right\|^{2}<C \varepsilon .
$$

The proof is complete.

Given a fixed even integer $N$, define $\left(\varphi_{k}\right)$ to be a sequence of functions generating the moment problem (8), i.e., $\mathrm{e}^{i \sqrt{\lambda_{k}} t}(|k| \leq N), \mathrm{e}^{i \sqrt{\lambda_{2 k-1}} t}(|k|>N)$, $\frac{\mathrm{e}^{i \sqrt{\lambda_{2 k}} t}-\mathrm{e}^{i \sqrt{\lambda_{2 k-1}} t}}{\sqrt{\lambda_{2 k}}-\sqrt{\lambda_{2 k-1}} t}(|k|>N), 1$ and $t$, with $\varphi_{0}(t)=t$, and define $\left(\varphi_{k}^{N}\right)$ to be a sequence of functions generating the moment problem (13), i.e., $\mathrm{e}^{i \sqrt{\lambda_{k}} t}(|k| \leq N), \mathrm{e}^{i \frac{2 k+1}{2} \pi t}$ $(|k|>N), t \mathrm{e}^{i \frac{2 k+1}{2} \pi t}(|k|>N), 1$ and $t$, with $\varphi_{0}^{N}(t)=t$. The family of sequences $\left(\varphi_{k}^{N}\right)$ approximates the initial one, $\left(\varphi_{k}\right)$, in the sense of the following theorem.

Theorem 4.1 For any $\varepsilon>0$ there exists $N_{0}$ such that for $N \geq N_{0}$ the families $\left(\varphi_{k}\right)$ and $\left(\varphi_{k}^{N}\right)$ are quadratically $\varepsilon$-close. 
Proof It suffices to prove that two series,

$$
\sum_{|n|>N}\left\|\mathrm{e}^{i \sqrt{\lambda_{2 n-1}} t}-\mathrm{e}^{i \frac{2 n-1}{2} \pi t}\right\|^{2}
$$

and

$$
\sum_{|n|>N}\left\|\frac{\mathrm{e}^{i \sqrt{\lambda_{2 n}} t}-\mathrm{e}^{i \sqrt{\lambda_{2 n-1}} t}}{\sqrt{\lambda_{2 n}} t-\sqrt{\lambda_{2 n-1}} t}-t \mathrm{e}^{i \frac{2 n+1}{2} \pi t}\right\|^{2},
$$

should be as small as needed for sufficiently large $N$. We know that $\varepsilon_{2 n-1}=\frac{2 n-1}{2} \pi-$ $\sqrt{\lambda_{2 n-1}}=\mathcal{O}\left(\frac{1}{n}\right)$ by (7) and therefore we can rewrite the $n$-th term of the first series as

$$
\begin{aligned}
\left\|\mathrm{e}^{i \sqrt{\lambda_{2 n-1}} t}-\mathrm{e}^{i \frac{2 n-1}{2} \pi t}\right\|^{2} & =\left\|\mathrm{e}^{i\left(n \pi-\varepsilon_{2 n-1}\right) t}-\mathrm{e}^{i n \pi t}\right\|^{2} \\
& =2 \int_{0}^{T} \mathrm{~d} t-2 \int_{0}^{T} \cos \left(\varepsilon_{2 n-1} t\right) \mathrm{d} t=\mathcal{O}\left(\frac{1}{n^{2}}\right),
\end{aligned}
$$

which means that for an arbitrary $\varepsilon>0$ we can find such a large $N_{1}$ that for any $N>N_{1}$ the following inequality holds:

$$
\sum_{|n|>N}\left\|\mathrm{e}^{i \sqrt{\lambda_{2 n-1}} t}-\mathrm{e}^{i \frac{2 n-1}{2} \pi t}\right\|^{2}<\frac{\varepsilon}{2} .
$$

Using the same method, one can see that there exists such a large $N_{2}$ that for any $N>N_{2}$,

$$
\sum_{|n|>N}\left\|\frac{\mathrm{e}^{i \sqrt{\lambda_{2 n}} t}-\mathrm{e}^{i \sqrt{\lambda_{2 n-1}} t}}{\sqrt{\lambda_{2 n}} t-\sqrt{\lambda_{2 n-1}} t}-t \mathrm{e}^{i \frac{2 n+1}{2} \pi t}\right\|^{2}<\frac{\varepsilon}{2} .
$$

Hence we obtain

$$
\sum_{k}\left\|\varphi_{k}-\varphi_{k}^{N}\right\|^{2}<\varepsilon
$$

for any $N>\max \left\{N_{1}, N_{2}\right\}$ which completes the proof.

Lemma 4.1 implies that not only the families $\left(\varphi_{k}\right)$ and $\left(\varphi_{k}^{N}\right)$ are quadratically $\varepsilon$ close, but their biorthogonal families $\left(\psi_{k}\right)$ and $\left(\psi_{k}^{N}\right)$ are also quadratically $\varepsilon$-close. Now we are ready to state the following 
Theorem 4.2 Let

$$
u_{N}:=\sum_{k}\left\langle u_{N}, \varphi_{k}^{N}\right\rangle \psi_{k}^{N}, \quad u_{0}:=\sum_{k}\left\langle u_{0}, \varphi_{k}\right\rangle \psi_{k},
$$

where $\varphi_{k}^{N}, \psi_{k}^{N}, \varphi_{k}$ and $\psi_{k}$ are defined above. Then

$$
\lim _{N \rightarrow \infty}\left\|u_{N}-u_{0}\right\|=0
$$

In consequence, the sequence of optimal solutions of moment problems (13) converges to the optimal solution of a moment problem (6).

Proof Define $\left(\psi_{\xi}\right):=\left(\psi_{k}\right) \cup\left(x_{k}\right)$ and $\left(\psi_{\xi}^{N}\right):=\left(\psi_{k}^{N}\right) \cup\left(P x_{k}\right)$. Because $\left(\psi_{\xi}\right)$ and $\left(\psi_{\xi}^{N}\right)$ are Riesz bases in $H=L^{2}[0, T]$ we may write

$$
u_{N}=\sum_{\xi}\left\langle u_{N}, \varphi_{\xi}^{N}\right\rangle \psi_{\xi}^{N}, \quad u_{0}=\sum_{\xi}\left\langle u_{0}, \varphi_{\xi}\right\rangle \psi_{\xi}
$$

Let $\varepsilon>0$ be given. By Theorem 4.1 there exists $N_{1}$ such that for any $N>N_{1}$ the families $\left(\varphi_{k}\right)$ and $\left(\varphi_{k}^{N}\right)$ are quadratically $\left(\frac{\varepsilon^{2}}{2 \theta_{T}^{2}}\right)$-close, from Lemma 4.1 we see that the families $\left(\psi_{k}\right)$ and $\left(\psi_{k}^{N}\right)$ are also quadratically $\left(\frac{\varepsilon^{2}}{2 \theta_{T}^{2}}\right)$-close. Lemma 4.2 implies that there exists $N_{2}$ such that for any $N>N_{2}$ the families $\left(x_{k}\right)$ and $\left(P x_{k}\right)$ are quadratically $\left(\frac{\varepsilon^{2}}{2 \theta_{T}^{2}}\right)$-close, too, therefore we see that the inequality

$$
\sum_{n}\left\|\psi_{n}^{N}-\psi_{n}\right\|^{2}<\frac{\varepsilon^{2}}{\theta_{T}^{2}}
$$

holds for $N>N_{0}=\max \left\{N_{1}, N_{2}\right\}$, which in particular means that

$$
\left\|\psi_{0}^{N}-\psi_{0}\right\|<\frac{\varepsilon}{\left|\theta_{T}\right|}
$$

Then for $N>N_{0}$ we have

$$
\begin{aligned}
\left\|u_{N}-u_{0}\right\| & =\left\|\sum_{n}\left\langle u_{N}, \varphi_{n}^{N}\right\rangle \psi_{n}^{N}-\sum_{n}\left\langle u_{0}, \varphi_{n}\right\rangle \psi_{n}\right\| \\
& =\left\|\theta_{T}\left(\psi_{0}^{N}-\psi_{0}\right)\right\|<\varepsilon .
\end{aligned}
$$

Therefore $\left(u_{N}\right)$ converges to $u_{0}$. 
We have shown that $\lim _{N \rightarrow \infty}\left\|u_{N}-u_{0}\right\|=0$. Using the following theorem one can see that even a stronger claim

$$
\lim _{N \rightarrow \infty} N\left\|u_{N}-u_{0}\right\|=0
$$

holds. To this end observe that the proof of Theorem 4 .1 shows not only that the families $\left(\varphi_{k}\right)$ and $\left(\varphi_{k}^{N}\right)$ are quadratically $\varepsilon$-close, but their differences admit the following representation:

$$
\varphi_{k}(x)-\varphi_{k}^{N}(x)=\frac{1}{k} \varphi_{k}(x)\left(g_{k}(x)+\frac{1}{k} h_{k}(x)\right),
$$

where $\left(g_{k}\right)$ is a uniformly bounded family, that is $\left\|g_{k}(x)\right\| \leq M$. Now we can proceed with

Theorem 4.3 Let $u_{N}, u_{0}, \varphi_{k}, \psi_{k}, \varphi_{k}^{N}$ and $\psi_{k}^{N}$ be as in Theorem 4.2. Then we have not only $\lim _{N \rightarrow \infty}\left\|u_{N}-u_{0}\right\|=0$ as before, but even

$$
\lim _{N \rightarrow \infty} N\left\|u_{N}-u_{0}\right\|=0 .
$$

Proof For simplicity of the proof we assume that the family $\left(g_{k}\right)$ consists of one function only, $g_{k}(x)=g(x)$. From previous considerations we know that

$$
u_{N}=u_{0}+\sum_{k=N+1}^{\infty}\left\langle u_{N}, \varphi_{k}\right\rangle \psi_{k}+\sum_{m}\left\langle u_{N}, P x_{m}\right\rangle x_{m}
$$

because $u_{N}$ is a solution of (13), and

$$
u_{N}=\sum_{k=0}^{N} \alpha_{k} \varphi_{k}+\sum_{k=N+1}^{\infty} \alpha_{k}^{N} \varphi_{k}^{N}
$$

for some $\alpha_{k}, \alpha_{k}^{N}$,s, because we assumed that $u_{N}$ belongs to $\operatorname{cl}\left(\operatorname{Lin}\left\{\left(\varphi_{k}\right)\right\}\right)$. Thus we can write

$$
N\left(u_{N}-u_{0}\right)=N \sum_{k=N+1}^{\infty}\left\langle u_{N}, \varphi_{k}-\varphi_{k}^{N}\right\rangle \psi_{k}+N \sum_{k=N+1}^{\infty} \sum_{m}\left\langle\alpha_{k}^{N}\left(\varphi_{k}^{N}-\varphi_{k}\right), P x_{m}\right\rangle x_{m},
$$


where again we used the fact that $u_{N}$ is a solution of (13). We will estimate the first sum only, the second one can be done in a similar way. We have

$$
\begin{aligned}
N^{2}\left\|\sum_{k=N+1}^{\infty}\left\langle u_{N}, \varphi_{k}-\varphi_{k}^{N}\right\rangle \psi_{k}\right\|^{2} \leq & M_{1} \sum_{k=N+1}^{\infty} \frac{N^{2}}{k^{2}}\left|\left\langle u_{N}, g \varphi_{k}\right\rangle\right|^{2} \\
& +M_{1} \sum_{k=N+1}^{\infty} \frac{N^{2}}{k^{2}}\left|\left\langle u_{N}, \frac{1}{k} h_{k} \varphi_{k}\right\rangle\right|^{2} .
\end{aligned}
$$

Now we observe that both summands tend to zero (when $N \rightarrow \infty$ ), namely

$$
\begin{aligned}
\sum_{k=N+1}^{\infty} \frac{N^{2}}{k^{2}}\left|\left\langle u_{N}, g \varphi_{k}\right\rangle\right|^{2} & \leq \sum_{k=N+1}^{\infty}\left|\left\langle u_{N}, g \varphi_{k}\right\rangle\right|^{2} \\
& \leq \sum_{k=N+1}^{\infty}\left|\left\langle u_{0} \bar{g}, \varphi_{k}\right\rangle\right|^{2}+\sum_{k=N+1}^{\infty}\left|\left\langle\left(u_{N}-u_{0}\right) \bar{g}, \varphi_{k}\right\rangle\right|^{2} \\
& \leq \sum_{k=N+1}^{\infty}\left|\left\langle u_{0} \bar{g}, \varphi_{k}\right\rangle\right|^{2}+\left\|\left(u_{N}-u_{0}\right) \bar{g}\right\|^{2} \rightarrow 0
\end{aligned}
$$

because the rest of a convergent series (of coefficients of $u_{0} \bar{g}$ in basis $\left(\psi_{k}\right)$ ) tends to zero, and $u_{N} \rightarrow u_{0}$, and

$$
\begin{aligned}
\sum_{k=N+1}^{\infty} \frac{N^{2}}{k^{2}}\left|\left\langle u_{N}, \frac{1}{k} h_{k} \varphi_{k}\right\rangle\right|^{2} & \leq \sum_{k=N+1}^{\infty} \frac{1}{k^{2}}\left|\left\langle u_{N}, h_{k} \varphi_{k}\right\rangle\right|^{2} \\
& \leq\left\|u_{N}\right\| \sup \left\|h_{k}\right\| \sup \left\|\varphi_{k}\right\| \sum_{k=N+1}^{\infty} \frac{1}{k^{2}} \rightarrow 0
\end{aligned}
$$

by similar arguments. Summarizing we obtain that

$$
\lim _{N \rightarrow \infty} N\left\|u_{N}-u_{0}\right\|=0 .
$$

We have shown that the sequence of solutions $u_{N}$ of the approximate moment problems (13) is not only convergent to the solution $u_{0}$ of the original moment problem (6), but the speed of convergence is considerably fast, better than any general methods could provide without taking into account the essential, individual properties of the families appearing in the moment problem in question.

\section{Numerical Solution of an Approximated Moment Problem}

Now after establishing the fact that the original moment problem (6) can be approximated by another moment problem (13) we will try to find an equivalent formulation 
of the latter, but now in a form of a finite number of equations. This in the end will enable the numerical analysis of the problem of construction of the optimal control.

At first let us observe that the set

$$
S_{N}:=\left\{1, t, \mathrm{e}^{i \sqrt{\lambda_{n}} t}(|n| \leq N), \mathrm{e}^{i \frac{2 n+1}{2} \pi t}(|n|>N), t \mathrm{e}^{i \frac{2 n+1}{2} \pi t}(|n|>N)\right\}
$$

is an $\mathcal{L}$-basis of $L^{2}[0, T]=L^{2}[0,2 M]$, that is it is a Riesz basis in closure of its linear span $V=\operatorname{cl}\left(\operatorname{Lin} S_{N}\right)$. Any control $u \in L^{2}[0,2 M]$ can be written in the unique form $u=u_{1}+u_{2}$, where $u_{1} \in V$ and $u_{2} \in V^{\perp}$. Since we have $\|u\|^{2}=\left\|u_{1}\right\|^{2}+\left\|u_{2}\right\|^{2}$ then due to the nature of our moment problem (13), which is generated by elements of $S_{N}$, we see that the solution $u$ has the least norm (i.e., it fulfills (5)) if and only if $\left\|u_{2}\right\|=0$, that is if and only if $u \in V$. This allows us to express the (unique) optimal solution $u$ as

$$
u(t)=\sum_{|n| \leq N} \alpha_{n} \mathrm{e}^{i \sqrt{\lambda_{n}} t}+\sum_{|n|>N} \beta_{n} \mathrm{e}^{i \frac{2 n+1}{2} \pi t}+\sum_{|n|>N} \gamma_{n} t \mathrm{e}^{i \frac{2 n+1}{2} \pi t}+A+B t
$$

where $\alpha_{n}, \beta_{n}, \gamma_{n}, A, B \in \mathbb{C}$ are (unknown) constants. Further on we rewrite

$$
\sum_{|n|>N} \beta_{n} \mathrm{e}^{i \frac{2 n+1}{2} \pi t}=\mathrm{e}^{i \frac{\pi}{2} t} \zeta(t), \quad \sum_{|n|>N} \gamma_{n} t \mathrm{e}^{i \frac{2 n+1}{2} \pi t}=\mathrm{e}^{i \frac{\pi}{2} t} t \eta(t),
$$

where $\zeta, \eta \in L^{2}[0,2 M]$ are (unknown) functions periodic on [0,2]. Thus

$$
u(t)=\sum_{|n| \leq N} \alpha_{n} \mathrm{e}^{i \sqrt{\lambda_{n}} t}+\mathrm{e}^{i \frac{\pi}{2} t} \zeta(t)+\mathrm{e}^{i \frac{\pi}{2} t} t \eta(t)+A+B t
$$

Directly from the definitions of $\zeta, \eta$ we see that

$$
\begin{aligned}
& \int_{0}^{2} \zeta(t) \mathrm{e}^{i n \pi t} \mathrm{~d} t=0, \quad|n| \leq N \\
& \int_{0}^{2} \eta(t) \mathrm{e}^{i n \pi t}=0, \quad|n| \leq N .
\end{aligned}
$$

Now we want to change our time interval from $[0,2 M]$ to $[0,2]$, where selected summands of (14) are either periodic or close to periodic. To this end we define

$$
\widehat{f}(s):=\sum_{k=0}^{M-1} f(s+2 k), \quad s \in[0,2]
$$

for any $f \in L^{2}[0,2 M]$. In long formulas we will write $[f(s)]^{\wedge}$ instead of $\widehat{f}(s)$. Substituting (14) into second line of (13) and using (15) we obtain 


$$
\begin{aligned}
0= & \int_{0}^{2 M} \mathrm{e}^{i \frac{2 n+1}{2} \pi t} u(t) \mathrm{d} t=\sum_{k=0}^{M-1} \int_{2 k}^{2 k+2} \mathrm{e}^{i \frac{2 n+1}{2} \pi t} u(t) \mathrm{d} t \\
= & \int_{0}^{2} \mathrm{e}^{i n \pi s}\left[\mathrm { e } ^ { i \frac { \pi } { 2 } s } M \left(\frac{1}{M} \sum_{|n| \leq N} \alpha_{n}\left[(-1)^{k} \mathrm{e}^{i \sqrt{\lambda_{n}} s}\right]^{\wedge}\right.\right. \\
& \left.\left.+\mathrm{e}^{i \frac{\pi}{2} s}(\zeta(s)+s \eta(s)+(M-1) \eta(s))+\widehat{(-1)^{k}}(A+B s+(M-1) B)\right)\right] \mathrm{d} s
\end{aligned}
$$

for all $|n|>N$, therefore we can rewrite the expression in the square brackets as $\sum_{|p| \leq N} a_{p} \mathrm{e}^{i p \pi s}$ with some unknown constants $a_{p} \in \mathbb{C},|p| \leq N$. Proceeding the same way with third line of (13) we express a similar term as $\sum_{|p| \leq N} b_{p} \mathrm{e}^{i p \pi s}$ with some unknown constants $b_{p} \in \mathbb{C},|p| \leq N$. Using those we can derive formulas for $\zeta$ and $\eta$, namely

$$
\begin{aligned}
\eta(s)= & \frac{3}{M\left(M^{2}-1\right)}\left(-\sum_{|m| \leq N} \alpha_{m}\left[(-1)^{k} s \mathrm{e}^{i \sqrt{\lambda_{m}} s}\right]^{\wedge} \mathrm{e}^{\frac{-i \pi s}{2}}\right. \\
& +(M+s-1) \sum_{|m| \leq N} \alpha_{m}\left[(-1)^{k} \mathrm{e}^{i \sqrt{\lambda_{m}} s}\right]^{\wedge} \mathrm{e}^{\frac{-i \pi s}{2}} \\
& +A \mathrm{e}^{\frac{-i \pi}{2}}\left(-(-1)^{M-1}\left(M-\frac{1}{2}\right)+\frac{1}{2}+\frac{1-(-1)^{M}}{2}(M-1)\right) \\
& +B \mathrm{e}^{\frac{-i \pi s}{2}}\left(-\frac{1-(-1)^{M}}{2}-2 M(M-1) s-4(-M+1) \frac{M}{2}\right. \\
& \left.+(M+s-1)\left(\frac{1-(-1)^{M}}{2} s+(-1)^{M-1}\left(M-\frac{1}{2}\right)-\frac{1}{2}\right)\right) \\
& \left.+\sum_{|p| \leq N} b_{p} \mathrm{e}^{i p \pi s} \mathrm{e}^{-i \pi s}-\sum_{|p| \leq N} a_{p}(M+s-1) \mathrm{e}^{i p \pi s} \mathrm{e}^{-i s \pi}\right)
\end{aligned}
$$

and

$$
\begin{aligned}
\zeta(s)= & -\frac{1}{M} \sum_{|m| \leq N}\left[(-1)^{k} \mathrm{e}^{i \sqrt{\lambda_{m}} s}\right]^{\wedge} \mathrm{e}^{\frac{-i s \pi}{2}}-\frac{1}{M} A \frac{1-(-1)^{M}}{2} \mathrm{e}^{\frac{-i s \pi}{2}} \\
& -\frac{1}{M} B \mathrm{e}^{\frac{-i s \pi}{2}}\left(\frac{1-(-1)^{M}}{2} s+(-1)^{M-1}\left(M-\frac{1}{2}\right)-\frac{1}{2}\right) \\
& +\frac{1}{M} \sum_{|p| \leq N} a_{p} \mathrm{e}^{i p \pi s} \mathrm{e}^{-i s \pi}-(M+s-1) \eta(s) .
\end{aligned}
$$

Now using all above and the remaining (first, fourth and fifth) lines of (13) we finally obtain $6 N+2$ linear equations of the following form: 


$$
\begin{aligned}
\int_{0}^{2} \mathrm{e}^{-i n \pi s}( & -\sum_{|m| \leq N} \alpha_{m}\left[(-1)^{k} s \mathrm{e}^{i \sqrt{\lambda_{m}} s}\right]^{\wedge} \mathrm{e}^{\frac{-i \pi s}{2}} \\
& +\sum_{|m| \leq N} \alpha_{m}\left[(-1)^{k} \mathrm{e}^{i \sqrt{\lambda_{m}} s}\right]^{\wedge} \mathrm{e}^{\frac{-i \pi s}{2}}(M+s-1) \\
& \left.+A \mathrm{e}^{\frac{-i \pi}{2}}\left(-(-1)^{M-1}\left(M-\frac{1}{2}\right)+\frac{1}{2}+\frac{1-(-1)^{M}}{2}(M-1)\right)\right) \\
& +B \mathrm{e}^{\frac{-i \pi s}{2}}\left(-\frac{1-(-1)^{M}}{2}-2 M(M-1) s-4(-M+1) \frac{M}{2}\right. \\
& \left.+(M+s-1)\left(\frac{1-(-1)^{M}}{2} s+(-1)^{M-1}\left(M-\frac{1}{2}\right)-\frac{1}{2}\right)\right) \\
& \left.+\sum_{|p| \leq N} b_{p} \mathrm{e}^{i p \pi s} \mathrm{e}^{-i \pi s}\right) \mathrm{d} s=0, \quad|n| \leq N,
\end{aligned}
$$$$
\int_{0}^{2}\left(-\frac{1}{M} \sum_{|m| \leq N}\left[(-1)^{k} \mathrm{e}^{i \sqrt{\lambda_{m}} s}\right]^{\wedge} \mathrm{e}^{\frac{-i s \pi}{2}}-\frac{1}{M} A \frac{1-(-1)^{M}}{2} \mathrm{e}^{\frac{-i s \pi}{2}}\right.
$$$$
-\frac{1}{M} B \mathrm{e}^{\frac{-i s \pi}{2}}\left(\frac{1-(-1)^{M}}{2} s+(-1)^{M-1}\left(M-\frac{1}{2}\right)-\frac{1}{2}\right)
$$$$
\left.-(M+s-1) \eta(s)+\frac{1}{M} \sum_{|p| \leq N} a_{p} \mathrm{e}^{i p \pi s} \mathrm{e}^{-i s \pi}\right) \mathrm{d} s=0, \quad|n| \leq N,
$$

$$
\begin{aligned}
& \sum_{|m| \leq N} \alpha_{m} \int_{0}^{2 M} \mathrm{e}^{i\left(\sqrt{\lambda_{m}}+\sqrt{\lambda_{n}}\right) t} \mathrm{~d} t+\int_{0}^{2 M} \mathrm{e}^{i \frac{\pi}{2} t} \zeta(t) \mathrm{e}^{i \sqrt{\lambda_{n}} t} \mathrm{~d} t \\
& +\int_{0}^{2 M} \mathrm{e}^{i \frac{\pi}{2} t} t \eta(t) \mathrm{e}^{i \sqrt{\lambda_{n}} t} \mathrm{~d} t+A \int_{0}^{2 M} \mathrm{e}^{i \sqrt{\lambda_{n}} t} \mathrm{~d} t+B \int_{0}^{2 M} t \mathrm{e}^{i \sqrt{\lambda_{n}} t} \mathrm{~d} t=0, \quad|n| \leq N,
\end{aligned}
$$

$$
\begin{aligned}
& \sum_{\substack{|m| \leq N \\
=0}} \alpha_{m} \int_{0}^{2 M} \mathrm{e}^{i \sqrt{\lambda_{m}} t} \mathrm{~d} t+\int_{0}^{2 M} \mathrm{e}^{i \frac{\pi}{2} t} \zeta(t) \mathrm{d} t+\int_{0}^{2 M} \mathrm{e}^{i \frac{\pi}{2} t} t \eta(t) \mathrm{d} t+A 2 M+B 2 M^{2} \\
& =0
\end{aligned}
$$

and

$$
\begin{aligned}
& \sum_{|m| \leq N} \alpha_{m} \int_{0}^{2 M} t \mathrm{e}^{i \sqrt{\lambda_{m}} t} \mathrm{~d} t+\int_{0}^{2 M} \mathrm{e}^{i \frac{\pi}{2} t} t \zeta(t) \mathrm{d} t+\int_{0}^{2 M} \mathrm{e}^{i \frac{\pi}{2} t} t^{2} \eta(t) \mathrm{d} t \\
& \quad+A 2 M^{2}+B \frac{8}{3} M^{3}=\theta_{T} .
\end{aligned}
$$


After a careful investigation (which we do not present here due to a sheer complication of its form in the general case) one can easily eliminate $\zeta$ and $\eta$ from above equations and in the end obtain a Cramer system of $6 N+2$ linear equations with exactly $6 N+2$ unknown values, namely $\alpha_{m}(|m| \leq N), a_{p}(|p| \leq N), b_{p}(|p| \leq N), A$ and $B$.

This way we showed that solving the approximate optimal moment problem (13) can be reduced to solving a finite number of linear equations which in turn can be solved numerically. Note that entries of Cramer matrix of this system depend on the first $N$ eigenvalues $\lambda_{n}$, which also have to be found numerically, but using the analytic formulas from [2] that can be done with any required level of precision. Thus we reduced the infinite dimensional problem of optimal control (1)-(5) to solving a system of a finite number of linear equations.

Remark 5.1 One should notice that the method presented herein uses only one approximation step — replacing the moment problem (8) by a close one (13) — and the latter can be solved without any further discretization errors.

\section{Conclusions}

In the paper, we constructed the optimal control for a slowly rotating Timoshenko beam. Basing on the specific character of the moment problem, corresponding to our optimal control problem, we presented a new method of approximating the original problem by another, special moment problem. We also showed that our approximation converges to the optimal solution with essentially faster speed than the one obtained by the classical truncation method. In the end, using special periodicity properties of the family of functions appearing in the approximated moment problem, we presented a method of reducing the system of infinite number of equations to the equivalent Kramer system of a finite number of equations. The whole process of constructing the optimal control function uses only one approximation step-replacing the moment problem in question by a close one-and the latter can be solved without any further discretization errors.

The methods introduced in the present paper can be also used in analysis of different control problems for other models, which spectral functions are close to periodic, e.g., vibrating strings, beams, and plates.

Acknowledgments The work was partially supported by Polish National Science Centre Grant No. N N514 238438. The authors wish to express their gratitude to anonymous referees for their valuable comments and constructive suggestions, which helped to improve the presentation of the results.

Open Access This article is distributed under the terms of the Creative Commons Attribution License which permits any use, distribution, and reproduction in any medium, provided the original author(s) and the source are credited.

\section{References}

1. Korobov, V.I., Krabs, W., Sklyar, G.M.: On the solvability of trigonometric moment problems arising in the problem of controllability of rotating beams. Internat. Ser. Numer. Math. 139, 145-156 (2001) 
2. Krabs, W., Sklyar, G.M.: On the controllability of a slowly rotating Timoshenko beam. Z. Anal. Anw. 18, 437-448 (1999)

3. Russell, D.L.: Nonharmonic Fourier series in the control theory of distributed parameter systems. J. Math. Anal. Appl. 18, 542-560 (1967)

4. Gugat, M.: Controllability of a slowly rotating Timoshenko beam. ESAIM: COCV 6, 333-361 (2001)

5. Krabs, W., Sklyar, G.M., Wozniak, J.: On the set of reachable states in the problem of controllability of rotating Timoshenko beams. Z. Anal. Anw. 22(1), 215-228 (2003)

6. Sklyar, G.M., Wozniak, J.: Ullrich conditions and smoothness of reachable states of a rotating beam. J. Math. Anal. Appl. 354, 31-45 (2009)

7. Sklyar, G.M., Szkibiel, G.: Spectral properties of non-homogeneous Timoshenko beam and its rest to rest controllability. J. Math. Anal. Appl. 338, 1054-1069 (2008)

8. Korobov, V.I., Krabs, W., Sklyar, G.M.: Construction of the control realizing the rotation of a Timoshenko beam. J. Optim. Theory Appl. 197, 51-68 (2000)

9. Gugat, M., Leugering, G., Sklyar, G.M.: Lp-optimal boundary control for the wave equation. SIAM J. Control Optim. 44, 49-74 (2005)

10. Gröchenig K., Strohmer T.: Numerical and theoretical aspects of nonuniform sampling of band-limited images. In: Nonuniform Sampling, Theory and Practice. Inform. Technol. Trans. Process. Storage, pp. 283-324. Kluwer Aademic/Plenum Publishers, New York (2001).

11. Strohmer, T.: Numerical analysis of the non-uniform sampling problem. J. Comput. Appl. Math. 122, 297-316 (2000)

12. Sklyar, G.M., Szkibiel, G.: Approximation of extremal solution of non-Fourier moment problem and optimal control for non-homogeneous vibrating systems. J. Math. Anal. Appl. 387, 241-250 (2012)

13. Krabs, W.: Optimal control of processes governed by partial differential equations part ii: vibrations. ZOR 26, 63-86 (1982)

14. Krabs, W., Sklyar, G.M.: Controllability of Linear Vibrations. NOVA Science Publishers Inc., Huntington, NY (2002)

15. Krabs, W.: On Moment Theory and Controllability of One-Dimensional Vibrating Systems and Heating Processes. Lecture Notes in Control and Information Sciences, vol. 173. Springer, Berlin (1992).

16. Gohberg, I.C., Krein, M.G.: Introduction to the theory of linear non-selfadjoint operators. AMS Translation of Mathematical Monographs, vol. 18, AMS, Providence (1969).

17. Ullrich, D.: Divided differences and systems of nonharmonic Fourier series. Proc. Am. Soc. 80, 47-57 (1980) 